\title{
Raleio químico de floração com complemento de pós floração na macieira grupo 'gala'
}

\author{
José Luiz Petri ${ }^{1}$; Gabriel Berenhauser Leite ${ }^{2}$; Poliana Francescatto ${ }^{3}$; \\ Gentil Carneiro Gabardo ${ }^{4}$; André Amarildo Sezerino ${ }^{5}$
}

${ }^{1}$ Empresa de Pesquisa Agropecuária e Extensão Rural de Santa Catarina - EPAGRI, Estação Experimental de Caçador, Rua Abilio Franco, 1500, Caçador, SC, CEP 89500-000. E-mail:Petri@epagri.sc.gov.br

${ }^{2}$ Empresa de Pesquisa Agropecuária e Extensão Rural de Santa Catarina - EPAGRI, Rodovia Admar Gonzaga, 1347,

Itacorubi, Florianopolis, SC, CEP 88034-901. E-mail : gabriel@epagri.sc.gov.br

${ }^{3}$ Cornell's New York State Agricultural Experiment Station, Geneva/NY, United States of America. E.mail: pf246@cornell.edu

${ }^{4}$ Doutorando em Produçáo Vegetal, UDESC/Centro de Ciências Agroveterinárias, Lages SC. E-mail: ge.gabardo@gmail.com>

${ }^{5}$ Empresa de Pesquisa Agropecuária e Extensāo Rural de Santa Catarina - EPAGRI, Estaçāo Experimental de Caçador,

Rua Abilio Franco, 1500, Caçador, SC, CEP 89500-000. E-mail: andresezerino@epagri.sc.gov.br

ISSN 2448-0479

Resumo - O raleio químico da macieira tem sido considerado como o método mais eficiente para realizar esta prática, principalmente quando há aplicação da combinação de BA com Carbaryl . Contudo, com a suspensão do registro do Carbaryl, novas alternativas tem sido desenvolvidas visando o raleio químico da macieira em pós-floração, destacando-se o ANA (Ácido Naftaleno Acético), o Promalin e mais recentemente a Benziladenina (BA).O objetivo deste estudo foi avaliar a resposta da aplicaçáo de raleantes químicos de floração com complemento de pós-floração, no raleio químico da macieira do grupo 'Gala', utilizado-se o clone 'Lisgala'. O experimento foi conduzido em pomar comercial localizado no município de Caçador, SC (265' $\mathrm{S}$; 5058'W; $947 \mathrm{~m}$ de altitude) na safra 2012/2013 e 2013/2014, tendo como delineamento experimental blocos casualizados, com 7 tratamentos e 6 repetiçóes de uma planta por parcela.. $\mathrm{Na}$ ausência do Carbaryl,por ser um inseticida do grupo dos fosforados, os produtos Promalin e Maxcel' são alternativas no raleio químico de macieiras do grupo 'Gala', os quais proporcionaram um efetivo raleio em floração e pós floração, promovendo o aumento na massa fresca média dos frutos.

Palavras-chave - Mallus domestica. Raleantes químicos. Benziladenina. Ácido giberélico. Produção.

Abstract - Chemical thinning is the most efficient method for fruit thinning because it can be done in the right time and in a short period, reducing labor costs and enhancing the return bloom. Due to the suspension of Carbaryl, new products have been explored to be used as post-bloom thinners on apples, such as NAA (naphthalene acetic acid), Promalin and more recently Benzyladenine (BA). This study aimed to evaluate the effect of double application of chemical thinners at bloom and post-bloom of 'Lisgala' apples. The trial was carried at two locations, in a commercial orchard located in Caçador, SC (Lat 26 $50^{\circ}$ 'S, Long 50 $58^{\prime} \mathrm{W}$ ) during 2012/13 and 2013/2014. In the absence of Carbaryl', Promalin and $\mathrm{Maxcel}^{\circ}$ are efficient alternatives for Gala apple thinning, contributing to larger fruit size.

Keywords - Mallus domestica. Chemical thinning. Benzyladenine. Fruit production.

\section{INTRODUÇÁO}

O raleio de frutos da macieira é uma das práticas culturais mais importantes para aumentar o calibre, melhorar a qualidade dos frutos, evitar a alternância de floração e manter a regularidade de produção (COSTA; DAL CIN; RAMINA, 2005; COSTA; BLANKE; WIDMER, 2013). Em anos com floração abundante e com condições ambientais favoráveis à polinização, a frutificação efetiva pode ser excessiva, fazendo com que haja excesso de frutos na planta, o que dificulta a obtençáo de frutos com maior calibre e, consequentemente, de maior valor comercial. Em macieiras, se todas as flores forem fecundadas e se de- 
senvolverem, a planta poderá não ter recursos fotossintéticos ou integridade estrutural para o adequado desenvolvimento e maturação dos frutos (GREENE; COSTA, 2013). Nestas condiçōes, o raleio é uma prática necessária e indispensável para se conseguir produçâo de frutos de qualidade e evitar a alternância de produção (COSTA; DAL CIN; RAMINA, 2005; COSTA; BLANKE; WIDME, 2013).

O raleio pode ser manual, mecânico ou químico. Dentre os métodos utilizados, o raleio químico tem sido considerado como o mais satisfatório, uma vez que pode ser realizado no momento mais adequado, em um curto período de tempo, diminuindo a mão de obra e com efeitos positivos no retorno da floraçáo (DENNIS, 2002).

Entre os raleantes químicos para a cultura da macieira destaca-se o ANA (Ácido Naftaleno Acético), o Carbaryl, o Ethephon, o $\mathrm{BA}+\mathrm{GA}_{4+7}$ e mais recentemente a Benziladenina (BA).

A BA é um composto do grupo das citocininas que atua na divisão celular, e por isso tem se mostrado efetiva no aumento do calibre dos frutos (MCLAUGHLIN; GREENE, 1984; GREENE, D. W. et al (1992); BYERS; CARBAUGH, 1991; WISMER et al., 1995). O efeito da BA no raleio da macieira é variável, o qual depende da época de aplicação e da concentração utilizada (GREENE et al., 1990; PETRI et al., 2013). Aplicações na floração ou em frutos com até $10 \mathrm{~mm}$ de diâmetro tendem a ser mais eficientes, assim como as concentraçôes mais elevadas. É considerada um bom produto raleante por apresentar baixo perfil toxicológico e simular a ação fisiológica da citocinina que é sintetizada nas plantas (YUAN; GREENE, 2000a, 2000b).

A combinação de BA com Carbaryl tem sido mais efetiva que a aplicação isolada, sendo considerada uma das mais eficientes combinaçóes para o raleio químico da macieira (BYERS, 2003). Contudo, por ser um inseticida de contato e ingestáo do grupo Metilcarbamato de naftila, o mesmo teve seu registro suspenso, devido a sua toxicidade e periculosidade ambiental e seu uso foi proibido no Brasil a partir de 2010. Desta forma, novas alternativas tem sido desenvolvidas visando o raleio químico da macieira em pós-floração (DORIGONI; LEZZER, 2007; CLEVER, 2007; MAAS, 2007).

O Promalin é uma mistura comercial de Benziladenina 1,8\% e Ácido Giberélico $\left(\mathrm{GA}_{4+7}\right)$ $1,8 \%$ que vem sendo utilizada como raleante de floraçẫo em locais onde a irregularidade da floração e frutificação devido a falta de frio hibernal resulta em um desenvolvimento de gemas de flores com anomalias anatômicas e morfológicas (THERON, 2013).

Maxcel $^{\circ}$ contém em sua formulação Benziladenina 2,0\%, sendo considerado um raleante de pós floração e vem sendo amplamente utilizado nas regiōes produtoras de maçãs.

O raleio de floração é importante quando ocorre abundante floração, característica dos clones do grupo 'Gala'. Entretanto, quando a macieira é cultivada em condiçôes onde seu requerimento em frio hibernal não é suficiente, ocorre uma floração irregular que se estende por um longo período, causando uma heterogeneidade no desenvolvimento dos seus estádios fenológicos. Nessas condiçôes, o raleio em pós floração é mais eficiente que o raleio de floração (FERNANDES, 2013).

O objetivo deste estudo foi avaliar a resposta da aplicação de raleantes químicos de floração com complemento de pós-floração, no raleio das macieiras 'Lisgala'.

\section{MATERIAIS E MÉTODOS/METODOLOGIA}

O experimento foi conduzido em pomar localizado no município de Caçador, SC, (26 50'S; 5058’W; $947 \mathrm{~m}$ acima do nível do mar) nas safras de 2012/2013 e 2013/2014. Foram utilizadas macieiras da cultivar Lisgala/M-26 (densidade de 2.500 plantas $\mathrm{ha}^{-1}$ ), que é um clone da cultivar Gala. As plantas foram conduzidas no sistema líder central e manejadas de acordo com recomendaçóes do sistema de produção integrada da macieira (EMPRESA DE PESQUISA AGROPECUÁRIA E EXTENSÃO RURAL DE SANTA CATARINA, 2006). O clima da região é classificado como "Cfb", temperado constantemente úmido, com verão ameno, segundo a classificação de Köeppen. Os dados de temperaturas médias máximas e medias mínimas e precipitação no período de avaliação da eficiência dos raleantes encontram-se dispostos na Figura 1.

O delineamento experimental utilizado foi de blocos casualizados, com sete tratamentos e seis repetiçôes, sendo a parcela constituída de uma planta. Os tratamentos e as datas de aplicaçấo estáo apresentados na Tabela 1.

Os produtos foram aplicados utilizando-se um pulverizador costal motorizado de $20 \mathrm{~L}$ com ponteira contendo três bicos D-S tipo leque, utilizando-se um volume de calda equivalente de $1000 \mathrm{~L}$ ha-1. 


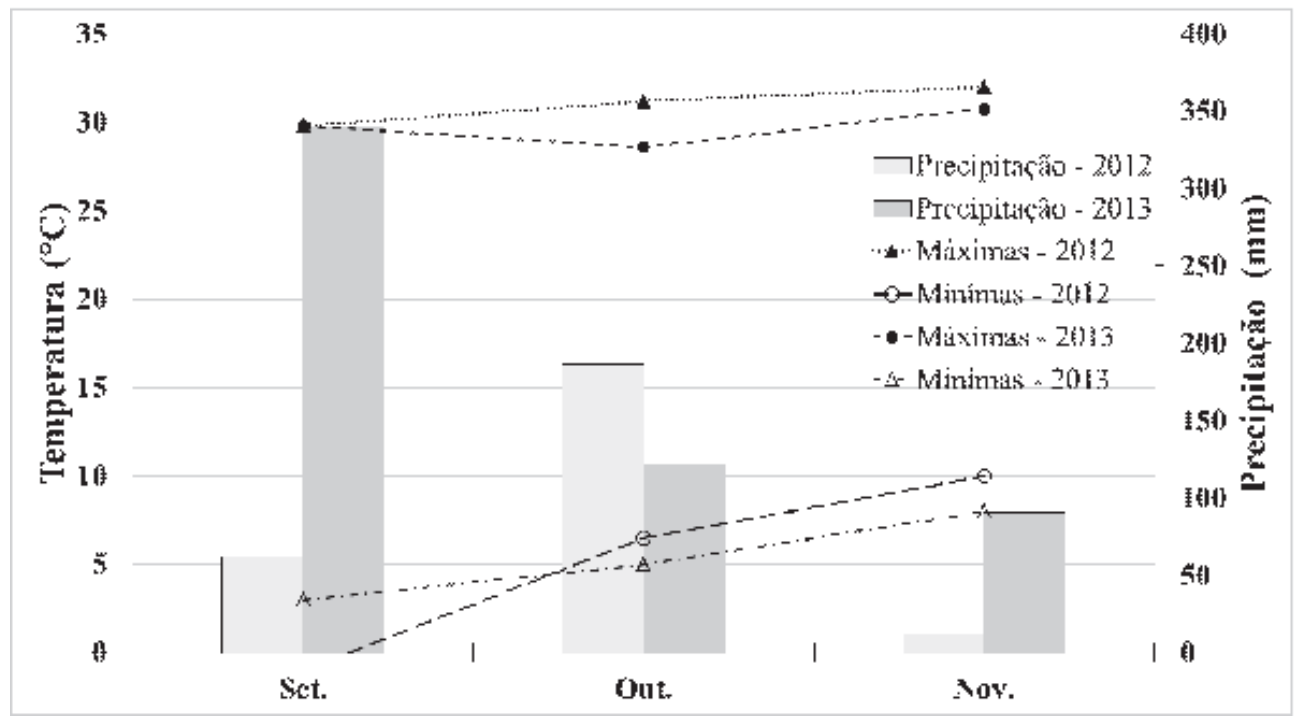

Figura 1 - Temperaturas médias máximas e médias mínimas diárias e precipitação diária durante o período de avaliação de eficiência de raleio da 'Lisgala'. Caçador, SC, 2016.

Tabela 1 - Composição dos tratamentos, doses e época das aplicaçôes dos raleantes químicos na macieira 'Lisgala' nas safras 2012/2013 e 2013/2014, Caçador, SC, 2016.

\begin{tabular}{|c|c|c|}
\hline \multirow{2}{*}{ Tratamentos } & \multicolumn{2}{|c|}{ Data de aplicação } \\
\hline & $2012 / 2013$ & $2013 / 2014$ \\
\hline Sem raleio - Controle & --- & --- \\
\hline Promalin 0,5L ha-1 PF + Promalin 0,5L ha ${ }^{-1} 7 \mathrm{DAPF}$ & $27 / 9$ e $05 / 10$ & $10 / 10$ e $17 / 10$ \\
\hline Promalin $0,5 \mathrm{~L} \mathrm{ha}^{-1} \mathrm{QP}+$ Promalin $^{\circ}$, 5L ha ${ }^{-1} 7 \mathrm{DAQP}$ & $11 / 10$ e $17 / 10$ & $17 / 10$ e $24 / 10$ \\
\hline Promalin $0,5 \mathrm{~L} \mathrm{ha}^{-1} \mathrm{PF}+$ Maxcel $^{\circ} 2,0 \mathrm{~L} \mathrm{ha}^{-1}-5-8 \mathrm{~mm}$ & $27 / 09$ e $17 / 10$ & $10 / 10$ e $22 / 10$ \\
\hline Promalin 0,5L ha ${ }^{-1} \mathrm{PF}+$ Maxcel $^{\circ} 2,0 \mathrm{~L} \mathrm{ha}^{-1}-10-15 \mathrm{~mm}$ & $27 / 09$ e $25 / 10$ & $10 / 10$ e $29 / 10$ \\
\hline Promalin 0,5 L ha ${ }^{-1} \mathrm{PF}+\left(\right.$ Promalin $^{\circ}$ 0,5 $\mathrm{L} \mathrm{ha}^{-1}+$ Maxcel $\left.^{\circ} 2,0 \mathrm{~L} \mathrm{ha}^{-1}-10-15 \mathrm{~mm}\right)$ & $27 / 09$ e $25 / 10$ & $10 / 10$ e $29 / 10$ \\
\hline Maxcel $^{\circ} 1,0 \mathrm{~L} \mathrm{ha}{ }^{-1} \mathrm{PF}+$ Maxcel $^{\circ} 2,0 \mathrm{~L} \mathrm{ha}^{-1}-5-8 \mathrm{~mm}$ & $27 / 09$ e $17 / 10$ & $10 / 10$ e $22 / 10$ \\
\hline
\end{tabular}

(1) Mistura comercial de Benziladenina 1,8\% e Ácido Giberélico (GA 4+7)1,8\%; ${ }^{(2)} \mathrm{PF}=\mathrm{Plena}$ floraçâo; ${ }^{(3)} \mathrm{DAPF}=\mathrm{Dias}$ após a plena floração; ${ }^{(4)}$ $\mathrm{QP}=\mathrm{Queda}$ de pétalas; ${ }^{(5)} \mathrm{DAQP}=\mathrm{Dias}$ após a queda das pétalas; ${ }^{(6)} 2 \%$ de $\mathrm{BA}$.

As variáveis avaliadas em ambas as safras foram: produção (frutos planta ${ }^{-1}$ e kg planta ${ }^{-1}$ ), a massa média fresca dos frutos, realizada através da colheita total dos frutos da planta, estimativa de produtividade $\left(\mathrm{t}\right.$ ha $\left.{ }^{-1}\right)$ obtida pela multiplicação da produção por planta e número de plantas por há ${ }^{-1} \mathrm{e}$ a distribuição dos frutos por classes de calibre, sendo os frutos de cada parcela classificados em três categorias (frutos menores que $55 \mathrm{~mm}$ de diâmetro, frutos com 55 a $65 \mathrm{~mm}$ de diâmetro, e frutos maiores que $65 \mathrm{~mm}$ de diâmetro), e calculada a sua porcentagem. A eficiência produtiva (frutos $\mathrm{cm}^{-2} \mathrm{e} \mathrm{kg} \mathrm{cm}^{-2}$ ) realizada através da medida da circunferência do tronco a $20 \mathrm{~cm}$ do solo e transformada em área $\mathrm{em} \mathrm{cm}^{2}$ sendo dividido o número e peso dos frutos pela área, a qual foi avaliada apenas em 2013/2014.

Nas variáveis que revelaram significância na análise de variância foi realizada a comparação de mé- dias pelo teste de Scott Knott a 5\% de probabilidade de erro, sendo utilizado o software Sisvar, versão 5.3 (FERREIRA, 2010).

\section{RESULTADOS E DISCUSSÁO}

A produção (kg planta ${ }^{-1}$ e frutos planta ${ }^{-1}$ ) apresentou diferenças significativas em ambos os anos, onde o tratamento sem raleio e Maxcel $^{\circ} 1,0$ $\mathrm{L} \mathrm{ha}{ }^{-1} \mathrm{PF}+$ Maxcel $^{\circ}$ 2,0 $\mathrm{L} \mathrm{ha}^{-1}$ 5-8 mm, foram os que apresentaram maior produção e número de frutos (Tabela 2). Já os tratamentos de Promalin $0,5 \mathrm{~L} \mathrm{ha}^{-1} \mathrm{PF}+$ Maxcel $^{\circ} 2,0 \mathrm{~L} \mathrm{ha}^{-1}$ em frutos com 5-8 mm e Promalin $0,5 \mathrm{~L} \mathrm{ha}^{-1} \mathrm{PF}+$ Maxcel $^{\circ} 2,0 \mathrm{~L}$ $\mathrm{ha}^{-1} \mathrm{em}$ frutos com $10-15 \mathrm{~mm}$ reduziram a frutificação tanto em kg planta ${ }^{-1}$ como em frutos plan$\mathrm{ta}^{-1}$, mostrando a ação raleante destes tratamentos em frutos até $15 \mathrm{~mm}$. 
Tabela 2 - Produção $\left(\mathrm{kg} \mathrm{planta}^{-1}\right.$ e frutos planta $\left.{ }^{-1}\right)$ de plantas de macieira submetidas a diferentes tratamentos com raleantes químicos, nas safras 2012/2013 e 2013/2014. Caçador, SC, 2016.

\begin{tabular}{|c|c|c|c|c|}
\hline \multirow{3}{*}{ Tratamentos } & \multicolumn{4}{|c|}{ Produção por planta } \\
\hline & \multicolumn{2}{|c|}{$2012 / 2013$} & \multicolumn{2}{|c|}{$2013 / 2014$} \\
\hline & kg & frutos & kg & frutos \\
\hline Sem raleio - Controle & $34,3 \mathrm{a}$ & $328,8 \mathrm{a}$ & $28,6 \mathrm{a}$ & $221,2 \mathrm{a}$ \\
\hline Promalin $0,5 \mathrm{~L} \mathrm{ha}^{-1} \mathrm{PF}+$ Promalin $0,5 \mathrm{~L} \mathrm{ha}^{-1} 7 \mathrm{DAPF}$ & $29,3 \mathrm{a}$ & $259,0 \mathrm{a}$ & $19,5 \mathrm{~b}$ & $143,5 \mathrm{~b}$ \\
\hline Promalin $0,5 \mathrm{~L} \mathrm{ha}^{-1} \mathrm{QP}+$ Promalin $^{\circ} 0,5 \mathrm{~L} \mathrm{ha}^{-1} 7 \mathrm{DAQP}$ & $26,5 \mathrm{a}$ & 276,7 a & $18,0 \mathrm{~b}$ & $131,2 \mathrm{~b}$ \\
\hline Promalin $0,5 \mathrm{~L} \mathrm{ha}^{-1} \mathrm{PF}+$ Maxcel $^{\circ} 2,0 \mathrm{~L} \mathrm{ha}^{-1}-5-8 \mathrm{~mm}$ & $24,8 \mathrm{~b}$ & $223,7 \mathrm{~b}$ & $17,2 \mathrm{~b}$ & $115,8 \mathrm{~b}$ \\
\hline Promalin $0,5 \mathrm{~L} \mathrm{ha}^{-1} \mathrm{PF}+$ Maxcel $^{\circ} 2,0 \mathrm{~L} \mathrm{ha}^{-1}$ frutos com $10-15 \mathrm{~mm}$ & $20,1 \mathrm{~b}$ & $181,5 \mathrm{~b}$ & $13,0 \mathrm{~b}$ & $90,2 \mathrm{~b}$ \\
\hline $\begin{array}{l}\text { Promalin } 0,5 \mathrm{~L} \mathrm{ha}^{-1} \mathrm{PF}+ \\
\text { (Promalin } 0,5 \mathrm{~L} \mathrm{ha}^{-1}+\text { Maxcel }^{\circ} 2,0 \mathrm{~L} \mathrm{ha}^{-1} \text { frutos com } 10-15 \mathrm{~mm} \text { ) }\end{array}$ & 30,0 a & $271,7 \mathrm{a}$ & $14,8 \mathrm{~b}$ & $102,7 \mathrm{~b}$ \\
\hline Maxcel $^{\circ} 1,0 \mathrm{~L} \mathrm{ha}^{-1} \mathrm{PF}+$ Maxcel $^{\circ} 2,0 \mathrm{~L} \mathrm{ha}^{-1}$ frutos com 5-8mm & $31,9 \mathrm{a}$ & $332,2 \mathrm{a}$ & $27,4 \mathrm{a}$ & $213,0 \mathrm{a}$ \\
\hline $\mathrm{CV}(\%)$ & 27,7 & 14,8 & 34,8 & 18,6 \\
\hline
\end{tabular}

Considerando que a produção estimada (Tabela 3 ) destes dois tratamentos em 2012/2013 foi superior a $50 \mathrm{t} \mathrm{ha}^{-1} \mathrm{e}$, em 2013/2014, superior a $32 \mathrm{t} \mathrm{ha}^{-1}$, pode-se considerar que os mesmos foram eficientes para a 'Lisgala' uma vez que reduziram a carga das plantas, mantendo uma produção aceitável. O número de frutos no tratamento de Promalin 0,5 $\mathrm{L} \mathrm{ha}^{-1} \mathrm{PF}+$ Maxcel 2,0 $\mathrm{L} \mathrm{ha}^{-1} \mathrm{em}$ frutos com 5-8 mm foi reduzido em $31,9 \%$ e $47,6 \%$ para 2012/2013 e 2013/2014, respectivamente e para o tratamento de Promalin 0,5
$\mathrm{L} \mathrm{ha}^{-1} \mathrm{PF}+$ Maxcel 2,0 $\mathrm{L} \mathrm{ha}^{-1}$ frutos 10-15 mm em $44,7 \%$ e $59,2 \%$ em 2012/2013 e 2013/2014, respectivamente. Deve-se destacar que no ciclo 2013/2014, a exceçáo do tratamento de Maxcel 1,0 L ha ${ }^{-1} \mathrm{PF}+$ Maxcel 2,0 $\mathrm{L} \mathrm{ha}^{-1}$ frutos com 5-8 mm, todos os demais reduziram a produção em $\mathrm{kg}_{\text {planta }}{ }^{-1}$, frutos planta $^{-1}$ e a produção estimada em relação ao tratamento sem raleio. Variaçôes na ação dos raleantes químicos entre anos pode estar relacionada as condiçóes ambientais ou até mesmo as reservas de carboidratos da planta.

Tabela 3 - Produção estimada $\left(\mathrm{t} \mathrm{ha}^{-1}\right)$ de plantas de macieira submetidas a diferentes tratamentos com raleantes químicos, nas safras 2011/2012 e 2012/2013. Caçador, SC, 2016.

\begin{tabular}{|c|c|c|}
\hline \multirow{2}{*}{ Tratamentos } & \multicolumn{2}{|c|}{ Produçáo estimada $\left(t_{\text {ha }}{ }^{-1}\right)$} \\
\hline & $2012 / 2013$ & $2013 / 2014$ \\
\hline Sem raleio- Controle & $85,7 \mathrm{a}$ & $71,5 \mathrm{a}$ \\
\hline 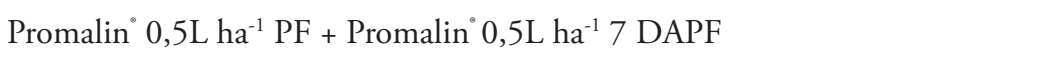 & 73,3 a & $48,7 \mathrm{~b}$ \\
\hline Promalin 0,5L ha ${ }^{-1} \mathrm{QP}+$ Promalin $^{\circ}, 5 \mathrm{~L} \mathrm{ha}^{-1} 7 \mathrm{DAQP}$ & $66,2 \mathrm{~b}$ & $45,0 \mathrm{~b}$ \\
\hline Promalin $0,5 \mathrm{~L} \mathrm{ha}^{-1} \mathrm{PF}+$ Maxcel $^{\circ} 2,0 \mathrm{~L} \mathrm{ha}{ }^{-1}-5-8 \mathrm{~mm}$ & $61,9 \mathrm{~b}$ & $43,0 \mathrm{~b}$ \\
\hline Promalin $0,5 \mathrm{~L} \mathrm{ha}^{-1} \mathrm{PF}+$ Maxcel $^{\circ} 2,0 \mathrm{~L} \mathrm{ha}{ }^{-1}-10-15 \mathrm{~mm}$ & $50,2 \mathrm{~b}$ & $32,5 \mathrm{~b}$ \\
\hline Promalin 0,5 $\mathrm{L} \mathrm{ha}^{-1} \mathrm{PF}+\left(\right.$ Promalin $^{\circ} 0,5 \mathrm{~L} \mathrm{ha}^{-1}+$ Maxcel $\left.^{\circ} 2,0 \mathrm{~L} \mathrm{ha}^{-1}-10-15 \mathrm{~mm}\right)$ & 75,0 a & $37,0 \mathrm{~b}$ \\
\hline Maxcel $^{\circ}$ 1,0L ha ${ }^{-1} \mathrm{PF}+$ Maxcel $^{\circ} 2,0 \mathrm{~L} \mathrm{ha}^{-1} 5-8 \mathrm{~mm}$ & 79,9 a & $68,5 \mathrm{a}$ \\
\hline $\mathrm{CV} \%$ & 27,7 & 32,3 \\
\hline
\end{tabular}

Médias seguidas de mesma letra, nâo diferem-se entre si, pelo teste de Scott Knott a 5\% de probabilidade. DAPF - dias após a plena floração; $\mathbf{P F}$ - plena floração; QP - queda das pétalas; DAQP - dias após queda das pétalas.

A eficiência produtiva medida na safra 2013/ 2014, demonstra a açâo raleante, pois todos os tratamentos que envolveram Promalin ou Promalin + Maxcel reduziram a eficiência produtiva medida por $\mathrm{kg} \mathrm{cm}{ }^{-2}$ ou frutos $\mathrm{cm}^{-2}$ (Tabela 4). Estes resultados evidenciam a ação raleante de Promalin na floração ou queda de pétalas com complemento de Promalin ou Maxcel em pós floração. 
Tabela 4 - Eficiência produtiva $\left(\mathrm{kg} \mathrm{cm}^{-2}\right.$ e frutos $\left.\mathrm{cm}^{-2}\right)$ de plantas de macieira submetidas a diferentes tratamentos com raleantes químicos, na safra 2013/2014. Caçador, SC, 2016.

\begin{tabular}{|c|c|c|}
\hline \multirow{2}{*}{ Tratamentos } & \multicolumn{2}{|c|}{ Eficiência produtiva } \\
\hline & $\mathrm{Kg} \mathrm{cm}^{-2}$ & Frutos $\mathrm{cm}^{-2}$ \\
\hline Sem raleio- Controle & $0,66 \mathrm{a}$ & $5,15 \mathrm{a}$ \\
\hline Promalin $0,5 \mathrm{~L} \mathrm{ha}^{-1} \mathrm{PF}+$ Promalin $0,5 \mathrm{~L} \mathrm{ha}^{-1} 7 \mathrm{DAPF}$ & $0,39 \mathrm{~b}$ & $2,84 \mathrm{~b}$ \\
\hline Promalin $0,5 \mathrm{~L} \mathrm{ha}^{-1} \mathrm{QP}+$ Promalin $0,5 \mathrm{~L} \mathrm{ha}^{-1} 7 \mathrm{DAQP}$ & $0,51 \mathrm{~b}$ & $3,75 \mathrm{~b}$ \\
\hline Promalin $0,5 \mathrm{~L} \mathrm{ha}^{-1} \mathrm{PF}+$ Maxcel $^{\circ} 2,0 \mathrm{~L} \mathrm{ha}^{-1}-5-8 \mathrm{~mm}$ & $0,39 \mathrm{~b}$ & $2,65 \mathrm{~b}$ \\
\hline Promalin 0,5L ha ${ }^{-1} \mathrm{PF}+$ Maxcel $^{\circ} 2,0 \mathrm{~L} \mathrm{ha}{ }^{-1}-10-15 \mathrm{~mm}$ & $0,31 \mathrm{~b}$ & $2,15 b$ \\
\hline Promalin 0,5L ha ${ }^{-1} \mathrm{PF}+\left(\right.$ Promalin $^{\circ} 0,5 \mathrm{~L} \mathrm{ha}^{-1}+$ Maxcel $\left.^{\circ} 2,0 \mathrm{~L} \mathrm{ha}^{-1}-10-15 \mathrm{~mm}\right)$ & $0,38 \mathrm{~b}$ & $2,72 \mathrm{~b}$ \\
\hline Maxcel $^{\circ}$ 1,0L hä ${ }^{-1} \mathrm{PF}+$ Maxcel $^{\circ} 2,0 \mathrm{~L} \mathrm{ha}^{-1} 5-8 \mathrm{~mm}$ & $0,72 \mathrm{a}$ & $5,58 \mathrm{a}$ \\
\hline $\mathrm{CV} \%$ & 29,5 & 31,3 \\
\hline
\end{tabular}

Médias seguidas de mesma letra, não diferem-se entre si, pelo teste de Scott Knott a 5\% de probabilidade. DAPF - dias após a plena floraçấo; $\mathbf{P F}$ - plena floração; QP - queda das pétalas; DAQP - dias após queda das pétalas.

A massa fresca média dos frutos foi significativamente diferente entre tratamentos, onde Promalin ${ }^{\circ}$ $0,5 \mathrm{~L} \mathrm{ha}^{-1} \mathrm{PF}+$ Promalin $^{\circ} 0,5 \mathrm{~L} \mathrm{ha}^{-1} 7 \mathrm{DAPF}$, Promalin 0,5 L ha ${ }^{-1} \mathrm{PF}+$ Maxcel $^{\circ} 2,0 \mathrm{~L} \mathrm{ha}^{-1}-5-8 \mathrm{~mm}$, Promalin 0,5 L ha- $\mathrm{PF}+$ Maxcel $^{\circ} 2,0 \mathrm{~L} \mathrm{ha}^{-1}-10-15$ mm e Promalin 0,5 L ha ${ }^{-1} \mathrm{PF}+$ (Promalin 0,5 L ha ${ }^{-1}$ + Maxcel $^{\circ}$ 2,0 L ha ${ }^{-1}-10-15 \mathrm{~mm}$ ) foram superiores ao tratamento testemunha e demais tratamentos em ambos os anos estudados (Tabela 5). Em 2012/2013 o aumento em relaçáo ao tratamento sem raleio variou de $6,3 \%$ a $8,7 \%$ e em $2013 / 2014$ foi de $5,9 \%$ a $14,3 \%$. Embora a produção por planta tenha sido superior no tratamento testemunha o que influencia a massa média dos frutos, as produtividades estimadas por hectare são consideradas adequadas mesmo nos tratamentos que aumentaram a massa média dos frutos. Segundo Costa (2013), um dos principais ob- jetivos do raleio químico é aumentar a massa média dos frutos. Os resultados também confirmam estudos de Greene (2005), Yuan e Greene (2000b), em que com o uso de Promalin ou Benziladenina no raleio químico da macieira proporciona um aumento da massa média dos frutos. Segundo Byers e Carbaugh (1991) BA é um composto do grupo das citocininas que atua na divisão celular e por isto tem se mostrado efetivo no aumento do tamanho dos frutos. Tustin et al. (2012) comentam que certas práticas culturais, entre elas a remoção do excesso de frutificação é uma das principais ferramentas para aumentar o tamanho dos frutos. Os resultados da massa média dos frutos evidenciaram que a produtividade influencia no tamanho dos frutos, visto que na safra 2013/2014 onde a produção foi inferior a safra anterior houve um incremento no peso médio dos frutos.

Tabela 5 - Massa fresca média dos frutos $\left(\mathrm{g} \mathrm{fruto}^{-1}\right)$ de plantas de macieira submetidas a diferentes tratamentos com raleantes químicos, nas safras 2012/2013 e 2013/2014. Caçador, SC, 2016.

\begin{tabular}{|c|c|c|}
\hline \multirow{2}{*}{ Tratamentos } & \multicolumn{2}{|c|}{ Massa fresca média dos frutos (g.fruto ${ }^{-1}$ ) } \\
\hline & $2012 / 2013$ & $2013 / 2014$ \\
\hline Sem raleio- Controle & $103,8 \mathrm{~b}$ & $129,8 \mathrm{~b}$ \\
\hline Promalin $0,5 \mathrm{~L} \mathrm{ha}^{-1} \mathrm{PF}+$ Promalin $^{\circ} 0,5 \mathrm{~L} \mathrm{ha}^{-1} 7 \mathrm{DAPF}$ & $112,8 \mathrm{a}$ & $137,5 \mathrm{a}$ \\
\hline Promalin 0,5L ha-1 $\mathrm{QP}+$ Promalin $^{\circ}, 5 \mathrm{~L} \mathrm{ha}^{-1} 7 \mathrm{DAQP}$ & $99,9 \mathrm{~b}$ & $139,3 \mathrm{a}$ \\
\hline Promalin $0,5 \mathrm{~L} \mathrm{ha}^{-1} \mathrm{PF}+$ Maxcel $^{\circ} 2,0 \mathrm{~L} \mathrm{ha}^{-1}-5-8 \mathrm{~mm}$ & $110,8 \mathrm{a}$ & $148,4 \mathrm{a}$ \\
\hline Promalin $0,5 \mathrm{~L} \mathrm{ha}{ }^{-1} \mathrm{PF}+$ Maxcel $^{\circ} 2,0 \mathrm{~L} \mathrm{ha}^{-1}-10-15 \mathrm{~mm}$ & $111,1 \mathrm{a}$ & $146,5 \mathrm{a}$ \\
\hline $\begin{array}{l}\text { Promalin } 0,5 \mathrm{~L} \mathrm{ha}^{-1} \mathrm{PF}+ \\
\text { (Promalin } 0,5 \mathrm{~L} \mathrm{ha}^{-1}+\text { Maxcel }^{\circ} \text { 2,0 } \mathrm{L} \mathrm{ha}^{-1}-10-15 \mathrm{~mm} \text { ) }\end{array}$ & $110,4 \mathrm{a}$ & 143,6 a \\
\hline Maxcel $^{\circ}$ 1,0L ha-1 PF + Maxcel $2,0 \mathrm{~L} \mathrm{ha}^{-1} 5-8 \mathrm{~mm}$ & $96,2 \mathrm{~b}$ & $129,4 \mathrm{~b}$ \\
\hline CV $(\%)$ & 8,6 & \\
\hline
\end{tabular}

Médias seguidas de mesma letra, não diferem-se entre si, pelo teste de Scott Knott a 5\% de probabilidade. DAPF - dias após a plena floração; $\mathbf{P F}$ - plena floração; QP - queda das pétalas; DAQP - dias após queda das pétalas. 
$\mathrm{Na}$ distribuição percentual dos frutos por classes de calibre, na safra de 2012/2013, houve uma redução no percentual de frutos de menor calibre nos tratamentos que aumentaram a massa média dos frutos, porém não houve diferenças significativas na classe de maior calibre e intermediária. Já na safra 2013/2014 houve um aumento do percentual de maior calibre e intermediaria e não havendo diferença na de menor calibre (Tabela 6).

Tabela 6 - Distribuição percentual nos calibres $>65,56-64$ e $<55$ de plantas de macieira submetidas a diferentes tratamentos com raleantes químicos, nas safras 2011/2012 e 2012/2013. Caçador, SC, 2016.

\begin{tabular}{|c|c|c|c|c|c|c|}
\hline \multirow{3}{*}{ Tratamentos } & \multicolumn{6}{|c|}{ Distribuiçáo percentual dos calibres } \\
\hline & \multicolumn{3}{|c|}{$2012 / 2013$} & \multicolumn{3}{|c|}{$2013 / 2014$} \\
\hline & $>65$ & $55-65$ & $<55$ & $>65$ & $55-65$ & $<55$ \\
\hline Sem raleio- Controle & $11,6^{\mathrm{ns}}$ & $51,6^{\mathrm{ns}}$ & 36,8 a & $24,4 \mathrm{~b}$ & $49,8 \mathrm{~b}$ & $25,8^{\mathrm{ns}}$ \\
\hline Promalin $0,5 \mathrm{~L} \mathrm{ha}^{-1} \mathrm{PF}+$ Promalin $^{\circ}, 5 \mathrm{~L} \mathrm{ha}^{-1} 7 \mathrm{DAPF}$ & 10,9 & 58,8 & $30,3 \mathrm{~b}$ & $35,5 \mathrm{a}$ & $48,7 \mathrm{~b}$ & 15,9 \\
\hline Promalin $0,5 \mathrm{~L} \mathrm{ha}^{-1} \mathrm{QP}+$ Promalin $0,5 \mathrm{~L} \mathrm{ha}^{-1} 7 \mathrm{DAQP}$ & 8,5 & 48,4 & 43,1 a & $24,7 \mathrm{~b}$ & $55,5 \mathrm{a}$ & 19,8 \\
\hline Promalin $0,5 \mathrm{~L} \mathrm{ha}^{-1} \mathrm{PF}+$ Maxcel $^{\circ} 2,0 \mathrm{~L} \mathrm{ha}^{-1}-5-8 \mathrm{~mm}$ & 16,4 & 59,5 & $24,2 \mathrm{~b}$ & 33,3 a & $45,8 \mathrm{~b}$ & 20,9 \\
\hline Promalin $0,5 \mathrm{~L} \mathrm{ha}^{-1} \mathrm{PF}+$ Maxcel $^{\circ} 2,0 \mathrm{~L} \mathrm{ha}{ }^{-1}-10-15 \mathrm{~mm}$ & 19,3 & 53,0 & $27,7 \mathrm{~b}$ & 37,1 a & $43,9 \mathrm{~b}$ & 19,0 \\
\hline $\begin{array}{l}\text { Promalin } 0,5 \mathrm{~L} \mathrm{ha}^{-1} \mathrm{PF}+ \\
\text { (Promalin } 0,5 \mathrm{~L} \mathrm{ha}^{-1}+\text { Maxcel }^{\circ} 2,0 \mathrm{~L} \mathrm{ha}^{-1}-10-15 \mathrm{~mm} \text { ) }\end{array}$ & 11,3 & 58,9 & $29,5 \mathrm{~b}$ & $32,8 \mathrm{a}$ & $50,0 \mathrm{~b}$ & 17,3 \\
\hline Maxcel $^{\circ} 1,0 \mathrm{~L} \mathrm{ha}{ }^{-1} \mathrm{PF}+$ Maxcel $^{\circ} 2,0 \mathrm{~L} \mathrm{ha}^{-1} 5-8 \mathrm{~mm}$ & 5,9 & 50,6 & 43,5 a & $17,5 \mathrm{~b}$ & $63,2 \mathrm{a}$ & 19,3 \\
\hline $\mathrm{CV} \%$ & 31,9 & 11,4 & 22,3 & 17,0 & 9,4 & 15,5 \\
\hline
\end{tabular}

\section{CONCLUSÁO}

Para o grupo 'Gala' e seus clones, os raleantes Promalin e Maxcel" são alternativas eficientes na floração e pós floração.

Promalin e Maxcel $^{\circ}$ proporcionam aumento da massa fresca média dos frutos.

\section{REFERÊNCIAS}

BYERS, R. E.; CARBOUGH, D. H. Effect of the chemical thinning sprays on the apples fruit set. HortTechnology, v.1, p. 41-48, 1991.

BYERS, R. E. Flower and fruit thinning and vegetative: fruiting balance. In: FERRE, D.C.; Warrington, I. J. (Eds.). Apples: botany, production and uses. Cambridge: CABI publishing, 2003. p. 409-436

CLEVER, M. A comparison of different thinning products applied to the apple variety 'Elstar Elshof on the lower Elbe region. Erwerbs Obstbau, v. 49, p.107-109. 2007.

COSTA, G. What's new in plant bioregulators? Acta Horticulturae. v. 998, p.27-36, 2013.

COSTA, G., DAL CIN, V., RAMINA, A. Pratical, phy- siological and molecular aspects of fruit abscission. Acta Horticulturae, v. 727, p. 301-310, 2005.

COSTA, G.; BLANKE, M.M.; WIDMER, A. Principles of thinning in fruit tree crops: needs and novelties. Acta Horticulturae, v. 998, p.17-26, 2013.

DENNIS, F.G. Mechanisms of action of apple thinning chemicals. HortScience, Alexandria, v.37, n.3, p.471474, 2002.

DORIGONI, A., LEZZER, P. Chemical thinning of apple with new compounds. Erwerbs-Obstbau, v. 49, p. 93-96, 2007.

EMPRESA DE PESQUISA AGROPECUÁRIA E EXTENSÃO RURAL DE SANTA CATARINA. A cultura da macieira. Florianópolis: EPAGRI, 2006. 743 p.

FERNANDES, C.; OLIVEIRA, C.M.; MOTA, M. Fruit thinning agents for apple cultivar 'Fuji': comparison among formulates. Acta Horticulturae. v. 998, p. 37-42, 2013.

FERREIRA, D. F. SISVAR: programa estatístico.

Versão 5.3 (Build 75). Lavras: Universidade Federal de Lavras, 2010.

GREENE, D. W. Chemicals, timing, and environmental factors involved in thinner efficacy on apple. HortScience, v. 37, p. 477-481, 2003. 
GREENE, D. W. Effects of repeated yearly application of chemical thinners on 'McIntosh' Apples. HortScience, v. 40, n.2, p.401-403, 2005.

GREENE, D. W. et al. Mode of action of benzyladenine when used as a chemical tinner. Journal of the American Society Horticultural Science, v. 117, p.775-779, 1992.

GREENE, D.W., AUTIO, W.R.; MILLER, P. Thinning activity of benzyladenine on several apple cultivars. Journal of the American Society Horticultural Science, v. 115, p. 390-400, 1990.

GREENE, D. W., COSTA, G. Fruit thinning in pomeand stone-fruit: State of the Art. Acta Horticulturae. v. 998, p.93-102, 2013.

MASS, F. 'Elstar' apples. Experiences with ammonium thiosulphate, calcium hydrioxide and benzyladenine. Erwerbs-Obstbau, v. 49, issue 3, p. 1001-105, 2007.

McLAUGHLIN, J.; DREENE, D.W. Effects of BA, GA $4+7$, and daminozide on fruit set, fruit quality, vegetative growth, flower initian, and flower quality of 'Golden Delicius' apple. Journal of the American Society Horticultural Science, v. 109, p.34-39, 1984.

PETRI, J. L. et al. Raleio químico em macieiras 'Fuji Suprema' e 'Lisgala'. Revista Brasileira de Fruticultura, Jaboticabal, v. 35, n. 1, p.170-182, 2013.

THERON, K. I. Chemical thinning of apple: South African Pespective. Acta Horticulturae. v. 998, p. 85-90, 2013.

TUSTIN, D.S. et al. Fruit set responses to changes in floral bud load - a new concept for crop load regulation. Acta Horticulturae. v. 932, p. 195-202, 2012..

WISMER, P.T.; PROCTOR, J. T. A.; ELFVING, D. C. Benzyladenine effects cell division and cell size during apple fruit thinning. Journal of the American Society Horticultural Science, v. 120, issue 5, p.802-807, 1995.

YUAN, R.; GREENE, D. W. Benzyladenine as a chemical thinner for McIntosh apples. I. Fruit thinning effects and associated relationships with photosynthesis, assimilate translocation, and nonstructural carbohydrates. Journal of the American Society for Horticultural Science, v.125, p. 169-176, 2000a.

YUAN, R.; GREENE, D. W. Benzyladenine as chemical thinner for 'McIntosh' apples. II. Effect of benzyladenine, bourse shoot tip removal, and life number on fruit retention. Journal American Society of Horticultural Science, v. 125, p. 177-182, 2000 b. 